\title{
NEAR CAPACITY RCQD CONSTELLATIONS FOR PAPR REDUCTION OF OFDM SYSTEMS
}

\author{
TarakArbi * Imen Nasr ${ }^{\dagger} \quad$ Benoit Geller * \\ * Department U2IS, ENSTA-Paris, Institut Polytechnique de Paris, Palaiseau, France \\ $\dagger$ Department COSIM, SUP'COM, University of Carthage, Ariana, Tunisia
}

\begin{abstract}
We investigate an optimized blind SeLected Mapping (SLM) algorithm to reduce the Peak-to-Average Power Ratio (PAPR) for Orthogonal Frequency Division Multiplexing (OFDM) systems with Signal Space Diversity (SSD). Several phase sequences based on two Rotated and Cyclically Q-Delayed (RCQD) constellations are used at the transmitter to considerably reduce the PAPR. The pair of RCQD constellations is selected so as to achieve the highest average mutual information for both the Coded Modulation (CM) and the Bit-Interleaved Coded Modulation (BICM) schemes. These constellations also allow, along with Hadamarad sequences, good blind detection performances with no spectral spoilage. Simulations totally confirm our theoretical results.
\end{abstract}

Index Terms - OFDM, PAPR, SeLected Mapping (SLM), Signal Space Diversity (SSD), Coded Modulation (CM), BitInterleaved Coded Modulation (BICM), Mutual Information.

\section{INTRODUCTION}

The efficiency and simplicity of OFDM modulation, over frequency selective channels, make it very attractive for high throughput communications. Nevertheless, the large PAPR of OFDM signals constitutes a serious limitation as it can result on both in-band distortion and out-of-band radiation. To remedy this issue, several solutions have been proposed such as SLM, Tone Reservation (TR), Partial Transmit Sequence, Active Constellation Extension (ACE) and Tone Injection $[1,2,3,4,5]$. The PAPR gain achieved by these methods is obtained at the price of either a loss of spectral efficiency or power efficiency, or a the cost of higher complexity [6].

Besides, the authors in [7] show that the transmission diversity over selective channels can be doubled, by the rotation of an ordinary constellation (i.e. QAM) symbol and the separate transmission of the interleaved In-phase (I) and Quadrature (Q) components over independent fading channels. Thanks to its efficiency [8, 9, 10], some standards have adopted this technique such as the DVB-T2 standard [11] which recommends some specific rotation angles to build the RCQD M-QAM signals [10, 11, 12]. Nevertheless, the choice of these angles is not unanimous in the literature
$[7,13,14,15,16,17]$. Besides, unlike the case of conventional QAM signals for which DVB-T2 provides two PAPR reduction methods (i.e. the ACE and the TR algorithms), only the TR method can be used when RCQD signals are used [11].

The authors in [16] recently proposed a blind SLM technique for OFDM systems with Signal Space Diversity (SSD); the transmitter having in its possession an alphabet of possible phase sequences, known by the receiver, chooses the phase sequence allowing the lowest PAPR. At the receiver side, the most likely sequence of rotation angles is estimated to avoid any spectral loss. This technique is based on two RCQD constellations that [16] proposes to select so as to jointly optimize the BER and the blind detection performance. In this paper, we elaborate on [16] to generalize the optimization process to the case of CM and BICM schemes [18, 21]. We also propose to use Hadamard sequences for the phase sequences generation as we show that the Hamming distance between these sequences impact considerably the system performance.

The rest of this paper is structured as follows. Section II discusses the system model. Section III presents the optimization framework and details our proposal. Simulation results are presented in Section IV. Section V concludes this paper.

\section{SYSTEM MODEL}

We now describe the system model depicted in Fig. 1. First, the user bits are channel encoded; the obtained codeword is then interleaved in compliance with the $\mathrm{CM}$ or BICM schemes. Then, every consecutive $N\left(\log _{2}(M)\right)$ bits are converted into $\mathrm{N}$ complex-valued $M-Q A M$ symbols as:

$$
s(n)=s_{1}(n)+j s_{2}(n),
$$

where $s_{1}(n)$ and $s_{2}(n)$ are respectively the I and Q components of $s(n)$. Instead of a unique rotation angle, as for the traditional RCQD modulation, the proposed transmitter possesses an alphabet of D possible N-length phase sequences: $\left\{P^{(d)}, d=0,1, \cdots, D-1\right\}$, where $P^{(d)}=$ $\left\{\theta_{0}^{(d)}, \theta_{1}^{(d)}, \cdots, \theta_{N-1}^{(d)}\right\}$. For every phase sequence $P^{(d)}$ and $n$ in $\{0,1, \cdots, N-1\}$, the transmitter first rotates the QAM symbol:

$$
x^{(d)}(n)=s(n) e^{j \theta_{n}^{(d)}}=x_{1}^{(d)}(n)+j x_{2}^{(d)}(n) .
$$




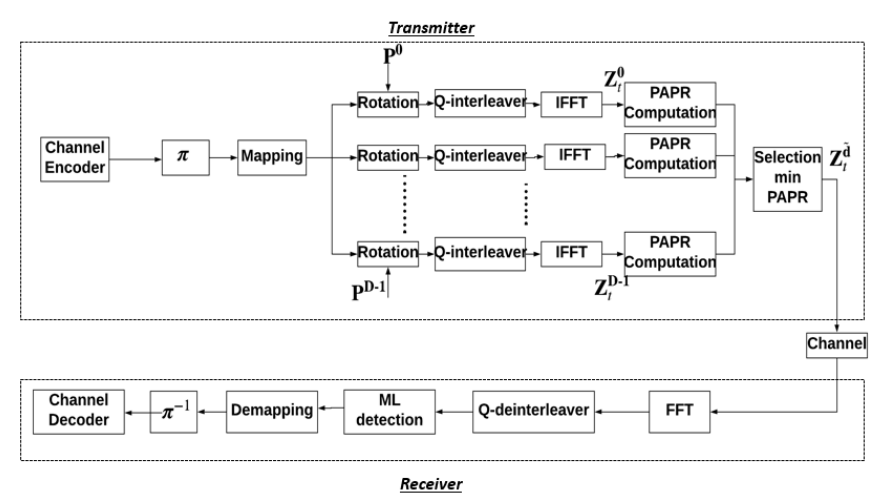

Fig. 1. System model; $\pi$ denotes interleaving at the symbol level (resp. at the bit level) for CM (resp. BICM).

Thereafter, the transmitter cyclically delays the $\mathrm{Q}$ component $x_{2}^{(d)}(n)$ so that the components $x_{1}^{(d)}(n)$ and $x_{2}^{(d)}(n)$ experiment independent channels:

$$
z_{f}^{(d)}(n)=x_{1}^{(d)}(n)+j x_{2}^{(d)}\left(n+\Delta_{T}\right),
$$

where $\Delta_{T}$ is an integer delay.

The transmitter then builds in parallel $D$ OFDM symbols:

$$
z_{t}^{(d)}(n)=\sum_{k=0}^{N-1} z_{f}^{(d)}(k) e^{\frac{2 \pi n k}{N}} .
$$

Before any transmission, for each phase sequence $P^{(d)}$, the transmitter computes the corresponding $P A P R(d)$ :

$$
\operatorname{PAPR}(d)=\frac{\max _{n=0, \cdots, N-1}\left|z_{t}^{(d)}(n)\right|^{2}}{\left.\mathbb{E}\left(\mid \boldsymbol{Z}_{t}^{(d)}\right)\right|^{2}},
$$

where $Z_{t}^{(d)}=\left(z_{t}^{(d)}(0), z_{t}^{(d)}(1), \cdots, z_{t}^{(d)}(N-1)\right)^{T}$ and $\mathbb{E}$ denotes the expectation. Finally, the transmitter selects $\tilde{d}$ as:

$$
\tilde{d}=\underset{d=0,1, \cdots, D-1}{\operatorname{argmin}} \operatorname{PAPR}(d),
$$

and delivers the corresponding OFDM symbol.

Assuming perfect synchronisation $[19,20]$, the received signal after Fast Fourier Transform (FFT) can be expressed as:

$$
y(n)=H(n) z_{f}^{(\tilde{d})}(n)+w(n),
$$

where $H$ is a Rayleigh random variable with unit variance and $w$ is a complex Gaussian noise of variance $2 N_{0}$. For a Rayleigh fading model with erasure events, the attenuation $h(n)$ is zero if an erasure event occurs, as defined in [11]. Then, the receiver reverses the interleaving as :

$$
\begin{aligned}
r(n) & =y_{1}(n)+j y_{2}\left(n-\Delta_{T}\right) \\
& =h_{1}(n) x_{1}^{(\tilde{d})}(n)+j h_{2}(n) x_{2}^{(\tilde{d})}(n)+v(n),
\end{aligned}
$$

where $v(n)$ and $h_{i}$ with $i \in\{1,2\}$ have the same statistical model characteristics as $w(n)$ and $H$ respectively. Thereafter, one can estimate the index $\tilde{d}$ using the Maximum Likelihood (ML) criterion as:

$$
\hat{d}=\underset{d=0,1, \cdots, D-1}{\operatorname{argmax}} P\left(R / P^{(d)}\right),
$$

where $\boldsymbol{R}=(r(0), r(1), \cdots, r(N-1))^{T}$.

Finally, the receiver computes the Log-Likelihood Ratio (LLR) for the mapped bits and, using the deinterleaved LLR values, the channel decoder then recovers the user bits.

\section{OPTIMIZATION FRAMEWORK}

With blind SLM techniques, the index $\tilde{d}$ needs to be estimated at the receiver side; hence, limiting the possible phase rotation angles to a couple of possibilities (i.e. $\left\{\theta_{n}^{(d)} \in\right.$ $\left.\left.\left\{\theta_{1}, \theta_{2}\right\}, \forall(d, n)\right\}\right)$ is beneficial as it substantially reduces the computational complexity.

\subsection{Index error probability analysis}

Let us define for $0 \leq d \leq D-1$ the phase code sequence $\boldsymbol{C}^{(d)}=\left\{c_{0}^{(d)}, c_{1}^{(d)} \cdots, c_{N-1}^{(\bar{d})}\right\}$ as:

$$
c_{n}^{(d)}=\left\{\begin{array}{l}
0 \text { if } \theta_{n}^{(d)}=\theta_{1} \\
1 \text { if } \theta_{n}^{(d)}=\theta_{2} .
\end{array}\right.
$$

Let $\mathbb{X}^{(d)}$ be the N-dimensional complex constellation corresponding to the phase code sequence $\boldsymbol{C}^{(d)}$. Using the union bound, the probability that the index $\hat{d}$ is decoded when the index $\tilde{d}$ is used at the transmitter can be upper bounded as:

$$
P\left(\boldsymbol{C}^{(\tilde{d})} \rightarrow \boldsymbol{C}^{(\hat{d})}\right) \leq \frac{1}{\mid \mathbb{X}^{(\tilde{d}) \mid}} \sum_{X \in \mathbb{X}^{(\tilde{d})}} P(\tilde{X}) \sum_{\hat{X} \in \mathbb{X}^{(\hat{d})}} P(\tilde{X} \rightarrow \hat{X}),
$$

where the independence between components leads to:

$$
P(\tilde{X} \rightarrow \hat{X})=\prod_{k=0}^{N-1} P(\tilde{X}(k) \rightarrow \hat{X}(k)),
$$

and $P(\tilde{X}(k) \rightarrow \hat{X}(k))$ can be upper bounded as follows [7]:

$$
P(\tilde{X}(k) \rightarrow \hat{X}(k)) \leq \frac{1}{2} \prod_{i=1}^{2} \frac{1}{1+\frac{\left(\tilde{X}_{i}(k)-\hat{X}_{i}(k)\right)^{2}}{8 N_{0}}} .
$$

Obtaining rotation angles by minimizing (11) is quite demanding in terms of complexity. Instead, one may rather minimize the average error probability $P_{\text {avg }}$ between points in $\mathbb{X}_{1}$ and in $\mathbb{X}_{2}$, where $\mathbb{X}_{i}$ is the constellation obtained by the rotation of the constellation $\mathbb{S}$ by the rotation angle $\theta_{i}$.

$$
P_{\text {avg }}\left(\theta_{1}, \theta_{2}\right)=\sum_{x \in \mathbb{X}_{1}} P(x) \sum_{\hat{x} \in \mathbb{X}_{2}} P(x \rightarrow \hat{x}),
$$


where $P(x \rightarrow \hat{x})$ is given by (13). Furthermore, (11) indicates that in order to minimize the index error probability, the Hamming distance between the phase code sequence $\boldsymbol{C}$ and $\boldsymbol{C}^{(\hat{d})}$ should be maximized. Thus, we here propose to use Hadamard sequences as they allow a high minimum Hamming distance between sets of sequences [23].

\subsection{Mutual information optimization}

Let us consider $\underline{X}_{i}$ and $\underline{\mathrm{R}}$, the random variable vectors that respectively represent the input and the output of the transmission channel, where $i$ can either be 1 or 2 according to the chosen rotation angle $\theta_{1}$ or $\theta_{2}$. The mutual information $I\left(\theta_{i}\right)=I\left(\underline{\mathrm{X}}_{i}, \underline{\mathrm{R}}\right)$ can be expressed as [22]:

$$
I\left(\theta_{i}\right)=H\left(\underline{\mathrm{X}}_{i}\right)-H\left(\underline{\mathrm{X}}_{i} \mid \underline{\mathrm{R}}\right) .
$$

The entropy $H\left(\underline{X}_{i}\right)$ is maximized for a uniformly distributed variable and then equals to $\log _{2} M$.

We now derive the expression of $H\left(\underline{X}_{i} \mid \underline{\mathrm{R}}\right)$ for CM and BICM schemes.

\subsubsection{The CM scheme}

The entropy $H\left(\underline{X}_{i} \mid \underline{\mathrm{R}}\right)$ can be expressed for a CM scheme scheme as [18]:

$H\left(\underline{\mathbf{X}}_{i} \mid \underline{\mathbf{R}}\right)=\int_{r \in \mathbf{C}} P(r) \int_{\left(h_{1}, h_{2}\right) \in \mathbf{R}_{+}^{2}} H\left(x \mid y, h_{1}, h_{2}\right) P\left(h_{1}, h_{2}\right) d h_{1} d h_{2} d r$

where:

$$
H\left(x \mid y, h_{1}, h_{2}\right)=-\sum_{x_{k} \in \epsilon_{i}} p\left(x_{k} \mid r, h_{1}, h_{2}\right) \log _{2}\left(p\left(x_{k} \mid r, h_{1}, h_{2}\right)\right) .
$$

\subsubsection{The BICM scheme}

Assuming ideal bit interleaving, the second term in (15) can be developed as $[18,21]$ :

$$
H\left(\underline{\mathrm{X}}_{i} \mid \underline{\mathrm{R}}\right)=\sum_{j=1}^{\log _{2} M} H\left(B_{i}^{j} \mid \underline{\mathrm{R}}\right)
$$

where $B_{i}^{j}$ denotes the $\mathrm{j}$-th binary channel; $H\left(B_{i}^{j} \mid \underline{\mathrm{R}}\right)$ can be expressed as:

$$
H\left(B_{i}^{j} \mid \underline{\mathbf{R}}\right)=-\int_{r \in \mathbb{C}} P(r) \int_{\substack{\left(h_{1}, h_{2}\right) \in \mathbb{R}_{+}^{2} \\ H}}\left(b_{j} \mid r, h_{1}, h_{2}\right)\left(h_{1}, h_{2}\right) d h_{1} d h_{2} d r,
$$

where:

$$
\begin{aligned}
& H\left(b_{j} \mid r, h_{1}, h_{2}\right)= \\
& \quad-\sum_{s=0,1} P\left(b_{j}=s \mid r, h_{1}, h_{2}\right) \log _{2}\left(P\left(b_{j}=s \mid r, h_{1}, h_{2}\right)\right),
\end{aligned}
$$

with:

$$
P\left(b_{j}=s \mid r, h_{1}, h_{2}\right)=\sum_{z \in \mathbb{X}_{i}^{j, s}} P\left(z \mid r, h_{1}, h_{2}\right)
$$

and $\mathbb{X}_{i}^{j, s}$ denotes the set of constellation points $\mathbb{X}_{i}$ whose $j$-th bit is $s$. Finally, $P\left(z \mid r, h_{1}, h_{2}\right)$ can be developed as:

$$
P\left(z \mid r, h_{1}, h_{2}\right)=\frac{P\left(r \mid z, h_{1}, h_{2}\right) P(z)}{\sum_{z^{\prime} \in \mathbb{X}_{i}} P\left(r \mid z^{\prime}, h_{1}, h_{2}\right) P\left(z^{\prime}\right)},
$$

with:

$$
P\left(r \mid z, h_{1}, h_{2}\right)=\frac{1}{2 \pi N_{0}} e^{\frac{-\left(r_{1}-h_{1} z_{1}\right)^{2}-\left(r_{2}-h_{2} z_{2}\right)^{2}}{2 N_{0}}} .
$$

\subsection{RCQD constellation preselection algorithm}

At the receiver side, a phase sequence error may be catastrophic for the system performance. We thus propose to first preselect all possible rotation angles couple for which the average error probability (14) is below a predefined threshold:

$$
\left.\left.\Psi_{\epsilon}=\left\{\left(\alpha_{1}, \alpha_{2}\right) \in\right]-\pi, \pi\right]^{2} \mid P_{a v g}\left(\alpha_{1}, \alpha_{2}\right)<\epsilon\right\},
$$

where $\epsilon$ refers to the threshold. A method to select a proper threshold is discussed in section 4.

As a second step, we need to find, among all the previously selected couples, the rotation angles $\left(\theta_{1}, \theta_{2}\right)$ that lead to the highest average mutual information as:

$$
\left(\theta_{1}, \theta_{2}\right)=\underset{\left(\alpha_{1}, \alpha_{2}\right) \in \Psi_{\epsilon}}{\operatorname{argmin}}\left(\frac{I\left(\alpha_{1}\right)+I\left(\alpha_{2}\right)}{2}\right),
$$

where the mutual information $I\left(\alpha_{i}\right)$ presented in (15) is computed using either (16) for the CM scheme or (18) for the BICM scheme.

\section{SIMULATION RESULTS}

Fig. 2 presents the Sequence Index Error Rate (SIER) obtained with random sequences and Hadamard sequences for rotation angles verifying $\epsilon_{1}=\min _{\left(\alpha_{1}, \alpha_{2}\right)}\left(P_{a v g}\left(\alpha_{1}, \alpha_{2}\right)\right)$, $\epsilon_{2}=2 \epsilon_{1}$ and $\epsilon_{4}=4 \epsilon_{1}$ (see (14)). First, it can observed that the SIER is rather satisfactory for low SNRs but that the performance greatly depends on the threshold; the threshold $\epsilon_{4}$ may lead to poor performances for a system operating at $E_{b} / N_{0}$ in the range of 10 to $14 \mathrm{~dB}$. Moreover, Hadamard sequences outperform random sequences in terms of SIER; thus, for the same SIER performance, the threshold $\epsilon$ can be relaxed with Hadamard sequences as this leads to a better optimization of the rotation angles in (25). Furthermore, Fig. 3 presents the average mutual information for the BICM 


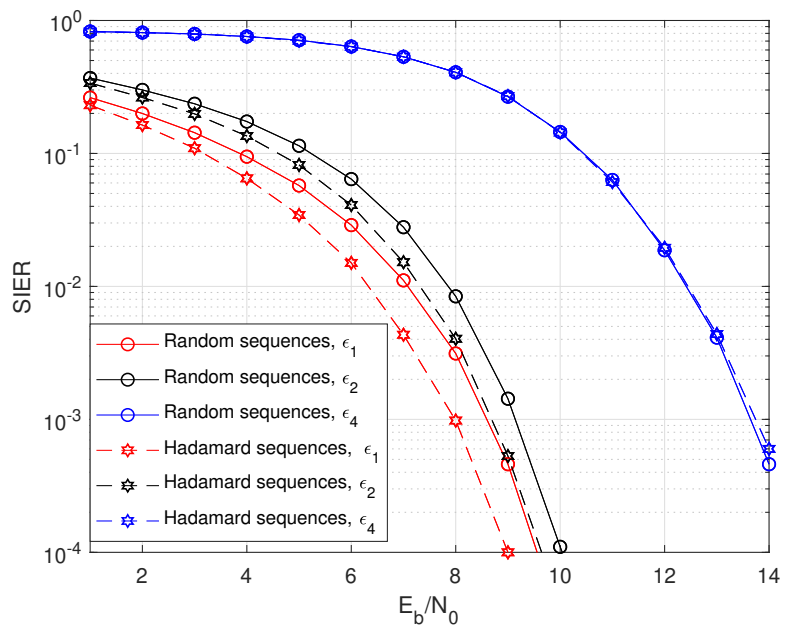

Fig. 2. SIER comparison between random and Hadamard sequences at different thresholds for the 64-QAM.

and CM models for the 64-QAM over Rayleigh fading channels at $E_{b} / N_{0}=13 \mathrm{~dB}$. It can first be observed that a poor choice of rotation angles may lead to a rate loss of up to 0.12 (resp. 0.16) bit/s/Hz for the BICM (resp. CM) schemes. In particular, the selected rotation angles with the threshold $\epsilon_{2}$ outperform the angle currently used in DVB-T2 for the 64QAM by about 0.02 (resp. 0.11) bit/s/Hz over the Rayleigh fading channel without erasure (resp. with $15 \%$ of erasure events). In addition, in contrast to [16], the rotation angle considerably depends on the system model. Indeed, the optimal rotation angles for the BICM model introduce a rate loss of about $0.09 \mathrm{bit} / \mathrm{s} / \mathrm{Hz}$ when used with the $\mathrm{CM}$ scheme.

Finally, for the 64-QAM constellation and $N=1024$, Fig. 4 compares the Complementary Cumulative Distribution Function (CCDF) of the PAPR corresponding to the TR algorithm defined in DVB-T2 [15] (with a clipping threshold of $7 \mathrm{~dB}$ and 16 iterations) with those of the proposed technique for $D=2,4,8,16$. It can be observed that for any $D$ larger than 2, our proposal achieves a better PAPR reduction than the TR algorithm. In addition, in contrast to the TR technique, our proposal avoids any spectral spoilage. Furthermore, compared to the original signal, the proposed method achieves a $3.7 \mathrm{~dB}$ gain for $D=16$, and even $4.9 \mathrm{~dB}$ when we combine the TR algorithm and our proposal for $D=8$.

\section{CONCLUSION}

A Blind SLM technique for OFDM systems with SSD was investigated. A theoretical framework was developed to optimally select the two RCQD constellations involved in the PAPR reduction algorithm. Simulations results confirm that our proposed technique tremendously reduces the PAPR with-
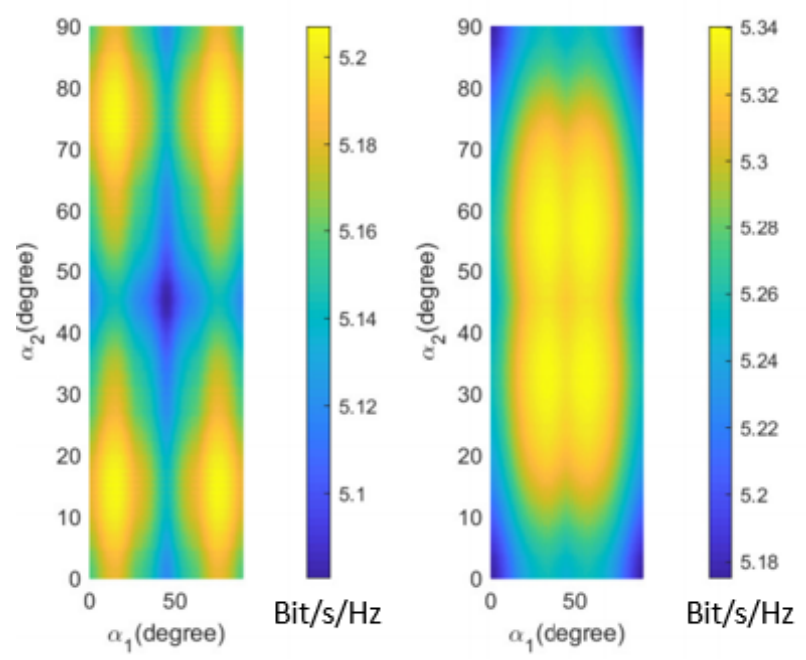

Fig. 3. Average mutual information as a function of the two rotation angles for the BICM (left) and the CM (right) models.

out any resource spoilage. In particular, it has been shown that the selection of a couple of rotation angles that minimizes the average pairwise error between the two RCQD constellations and the adoption of Hadamard sequences in the construction of phase sequences lead to robust blind detection performances. In addition, selecting rotated constellations by maximizing the average mutual information leads to a significant theoretical improvement compared to the solution currently used in DVB-T2 for both CM and BICM schemes, especially over fading channel with erasure. Therefore, our proposed technique is attractive, especially for systems with high spectral [24] and power efficiencies requirements.

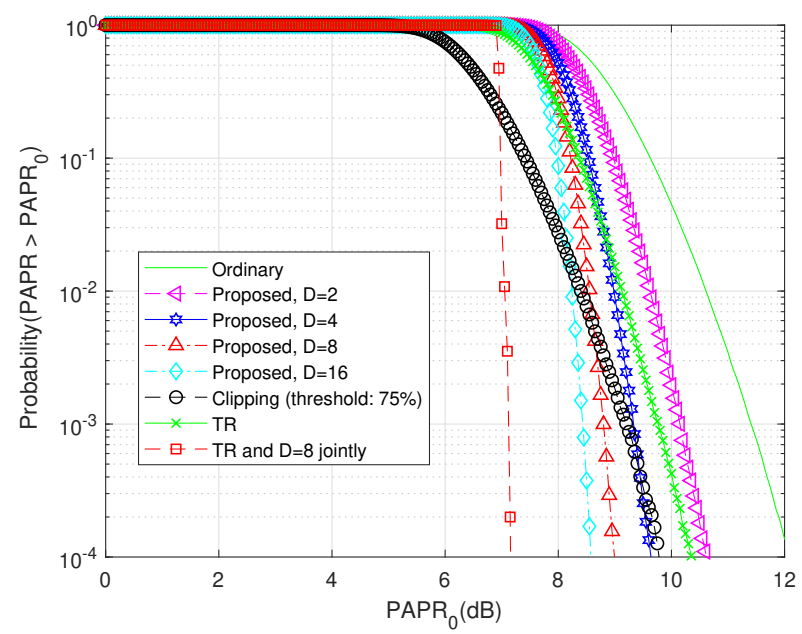

Fig. 4. PAPR reduction performance comparison between the considered methods for an OFDM system with 1024 subcarriers and the RCQD 64-QAM. 


\section{REFERENCES}

[1] Dae-Woon Lim, Jong-Seon No, Chi-Woo Lim and Habong Chung,"A new SLM OFDM scheme with low complexity for PAPR reduction,"IEEE Signal Process. Letters, 12 (2), pp. 93-96, Feb. 2005.

[2] S. S. K. C. Bulusu, M. Crussière, J. F. Hélard, R. Mounzer, Y. Nasser, O. Rousset, and A. Untersee, "QuasiOptimal Tone Reservation PAPR Reduction Algorithm for Next Generation Broadcasting Systems: A Performance/Complexity/Latency Tradeoff With Testbed Implementation," in IEEE Trans. on Broadcasting, 64 (4), pp. 883-899, Dec. 2018.

[3] X. Qi, Y. Li and H. Huang, "A Low Complexity PTS Scheme Based on Tree for PAPR Reduction," IEEE Comm. Letters, 16 (9), pp. 1486-1488, Sept. 2012.

[4] K. Bae, J. G. Andrews and E. J. Powers, "Adaptive active constellation extension algorithm for peak-toaverage ratio reduction in OFDM," in IEEE Communications Letters, 14 (1), pp. 39-41, January 2010.

[5] C. Tuna and D. L. Jones, "Tone injection with aggressive clipping projection for OFDM PAPR reduction," IEEE International Conference on Acoustics, Speech and Signal Processing, Dallas, 2010, pp. 3278-3281.

[6] D. Lim, S. Heo and J. No, "An overview of peak-toaverage power ratio reduction schemes for OFDM signals," Journal of Communications and Networks, 11 (3), pp. 229-239, June 2009.

[7] J. Boutros, and E. Viterbo, "Signal space diversity: a power- and bandwidth-efficient diversity technique for the Rayleigh fading channel", IEEE Trans. on Inform. Theory, 44 (4), pp. 1453-1467, July 1998.

[8] D. Gozálvez, J. J. Giménez, D. Gómez-Barquero and N. Cardona, "Rotated Constellations for Improved Time and Frequency Diversity in DVB-NGH," IEEE Transactions on Broadcasting, 59 (2), pp. 298-305, June 2013.

[9] C. Abdel Nour and C. Douillard, "Rotated QAM Constellations to Improve BICM Performance for DVB-T2," IEEE 10th Inter. Sym. on Spread Spectrum Techniques and Applications, Bologna, pp. 354-359, Aug. 2008.

[10] M. Li, C. A. Nour, C. Jego and C. Douillard, “ Design of rotated QAM mapper/demapper for the DVB-T2 standard," IEEE Workshop on Signal Processing Systems, Tampere, pp. 018-023, Nov. 2009.

[11] Implementation guidelines for a second generation digital terrestrial television broadcasting system (DVB-T2), European Telecom. Standard Institute ETSI TR 102831 V1.1.1, Oct. 2010.
[12] D. Perez-Calderon, V. Baena-Lecuyer, A. C. Oria, P. Lopez and J. G. Doblado, "Rotated constellation demapper for DVB-T2," Elec. Letters, 47 (1), pp. 31-32, Jan. 2011.

[13] M. Khormuji, U. Rizvi, G. Janssen, S. Silmane, "Rotation optimization for MPSK/MQAM signal constellation over Rayleigh fading channels", Proc. 10th IEEE Int. Conf. on Commun. Sys., Singapore, 2006, pp. 1-5.

[14] Q. Xie et al., "Coded modulation with signal space diversity," IEEE Trans. on Wireless Communication, 10 (2), pp. 660-669, Feb. 2011.

[15] A. Abdmouleh, E. Boutillon, L. Conde-Canencia, C. A. Nour, C. Douillard, "On Signal Space Diversity for non binary coded modulation schemes," IEEE 23rd Inter. Conf. on Telecomm.(ICT), Greece. pp. 1-5, 2016.

[16] T. Arbi and B. Geller, "Joint BER Optimization and Blind PAPR Reduction of OFDM Systems with Signal Space Diversity," IEEE Communications Letters, 23 (10), pp. 1866-1870, Oct. 2019.

[17] T. Arbi, B. Geller, J. Yang, C. Abdel Nour and O. Rioul, "Uniformly Projected RCQD QAM: A LowComplexity Signal Space Diversity Solution Over Fading Channels With or Without Erasures," IEEE Trans. on Broadcasting, vol. 64, no. 4, pp. 803-815, Dec. 2018.

[18] L. Szczecinski and A. Alvarado, "Bit-Interleaved Coded Modulation: Fundamentals, Analysis and Design,"John Wiley, Chichester, UK, 2015.

[19] I. Nasr, B. Geller, L. N. Atallah and S. Cherif, "Performance Study of a Near Maximum Likelihood CodeAided Timing Recovery Technique," IEEE Transactions on Signal Processing, 64 (3), pp. 799-811, Feb.2016.

[20] J. Yang, B. Geller and S. Bay, "Bayesian and Hybrid Cramér-Rao Bounds for the Carrier Recovery Under Dynamic Phase Uncertain Channels," IEEE Trans. on Signal Processing, 59 (2), pp. 667-680, Feb. 2011.

[21] G. Caire, G. Taricco and E. Biglieri, "Bit-interleaved coded modulation," IEEE Transactions on Information Theory, 44 (3), pp. 927-946, May 1998.

[22] T. M. Cover and J. A. Thomas, "Elements of Information Theory,"Wiley Series in Telecommunications and Signal Processing, 2006, New York, USA.

[23] J. Seberry and M. Yamada, "Hadamard matrices, Sequences, and Block Designs," John Wiley and Sons, 431560. 1992.

[24] C. Vanstraceele, B. Geller, J.P. Barbot, J.M. Brossier, "Block turbo codes for multicarrier local loop transmission," Proc. of IEEE 56th VTC Vehicular Technology Conference, pp. 1773-1776, Vancouver, Sept. 2002. 